\title{
Common-Mode Voltage Reduction in a VSI Inverter Applying Sequential Predictive Control
}

\author{
Duberney Murillo-Yarce, Marco Rivera, Carlos Restrepo, Javier Muñoz, Carlos Baier, Raúl Rodríguez, \\ Patrick Wheeler, Pericle Zanchetta and Galina Mirzaeva
}

\begin{abstract}
In the classical predictive control (CPC) implementation a cost function designed in terms of the control objectives and constants known as weighting factors must be minimized. Weighting factors are usually obtained by trial and error since there is no methodology to find the best factors, which also improve the controller performance. One possible solution is sequential predictive control (SPC) that proposes to eliminate weighting factors by sequential evaluation of simple cost functions. The contribution of this work is to analyze in detail the SPC performance and compare the results with a classic predictive current controller, in a VSI converter feeding an RL load where common-mode voltage is also reduced. Matlab/Simulink simulation results show that the SPC technique is able to reduce the common-mode voltage by half and still obtaining a balanced current system with an allowable THD, which is not feasible for the CPC technique. These findings demonstrate that SPC can improve results obtained with $\mathrm{CPC}$ without using weighting factors.
\end{abstract}

Keywords-Common-mode voltage reduction, current tracking, sequential predictive control, voltage source inverter.

\section{INTRODUCCIÓN}

$\mathbf{E}$ L control predictivo ha sido ampliamente aplicado en años recientes especialmente en electrónica de potencia [1]. Sus principales ventajas son simplicidad, fácil implementación y la posibilidad de incluir no linealidades y restricciones [2], [3]. En electrónica de potencia la técnica más utilizada es control predictivo de estados finitos basado en modelo, la cual utiliza los posibles estados de conmutación para definir la secuencia que optimiza el desempeño [4]. Esta técnica de control requiere el modelo matemático en tiempo discreto del convertidor de potencia para predecir los valores futuros de la variable de control para el conjunto de estados válidos. Estos resultados son evaluados en la funcion de costos para obtener el estado óptimo que será aplicado en el siguiente período de muestreo [5]. La función de costos es una expresión matemática diseñada en función de los objetivos de control. Particularmente para el convertidor VSI alimentando una carga

D. Murillo-Yarce, M. Rivera, C. Restrepo, J. Muñoz y C. Baier están con la Facultad de Ingeniería de la Universidad de Talca, Curicó, Chile, e-mail: duberney.murillo@utalca.cl,marcoriv@utalca.cl,crestrepo@utalca.cl, jamunoz@utalca.cl, cbaier@utalca.cl.

R. Rodríguez está con Universidad Tecnológica de Pereira, Pereira, Colombia, e-mail: raul.rodriguez@utp.edu.co.

P. Wheeler y P. Zanchetta están con Faculty of Engineering, University of Nottingham, Nottingham, UK-China, e-mail: pat.wheeler@nottingham.ac.uk, pericle.zanchetta@nottingham.ac.uk.

G. Mirzaeva está con School of Electrical Engineering and Computer Science University of Newcastle, Australia, e-mail: galina.mirzaeva@newcastle.edu.au.

978-0-7381-3333-1/21/\$31.00@2021IEEE
RL, el principal objetivo de control es el seguimiento de la corriente de salida. Pueden existir objetivos secundarios como la reducción del contenido armónico y el voltaje en modo común, la minimización de las pérdidas por conmutación y la regulación del voltaje del bus dc. Para funciones de costo con más de un objetivo de control es necesario incluir factores de peso de acuerdo a la teoría del control predictivo clásico (CPC). El valor de los factores de peso depende de prioridades previamente establecidas y del comportamiento deseado [6].

La sintonización de los factores de peso es un proceso complejo ya que no se ha establecido ningún procedimiento para encontrar los mejores valores. Los factores de peso usualmente se obtienen por ensayo y error [7]. Es posible realizar una mejor selección de los factores de peso basado en el conocimiento de la aplicación, conceptos físicos y relaciones entre variables. Una posible solución es la evaluación secuencial de funciones de costo simples. Así, los factores de peso no son requeridos. Esta es la característica fundamental del control predictivo secuencial (SPC) [8]. Por ejemplo, en el caso de dos objetivos de control todos los estados de conmutación son evaluados en la primera función de costos. Mientras que en la siguiente función de costos, sólo el subconjunto de estados que mejor minimizan la primera función son evaluados. El número de elementos de este subconjunto es un parámetro del algoritmo SPC. SPC es una nueva tendencia en control predictivo. Hay algunas publicaciones recientes sobre conversión ac y accionamientos de motores eléctricos. En conversión ac, el SPC ha sido aplicado a convertidores matriciales [7]. Por su parte, en accionamientos eléctricos se han utilizado convertidores de diodos limitadores de tres niveles ó NPC (neutral point clamped) [9], [10] y convertidores VSI [8], [11]. Una aplicación especial es una estructura de dos convertidores VSI utilizados para motores de inducción de bobinado abierto [12]. La contribución de este artículo es analizar de manera detallada el desempeño del controlador SPC y comparar los resultados con un controlador de corriente predictivo clásico para un convertidor VSI alimentando una carga RL, donde también se reduce el voltaje en modo común. Un balance entre los objetivos de control puede ser logrado fácilmente con la técnica SPC, lo que es bastante complejo para la técnica CPC. La estructura de este trabajo es la siguiente: en la Sección II se presenta el modelo matemático del sistema de potencia formado por el convertidor VSI y la carga RL. En la Sección III se analizan los controladores predictivos especialmente el SPC. Los resultados de simulación y la discusión se incluyen en las Secciones IV y V. Finalmente, en la Sección VI se presentan las conclusiones. 


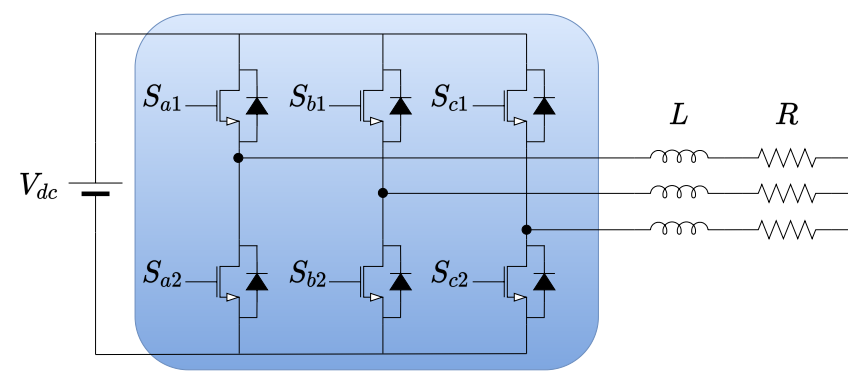

Fig. 1. Circuito de potencia del convertidor VSI alimentando una carga RL.

\section{MODELO MATEMÁTICO DEL CONVERTIDOR VSI}

\section{A. Convertidor VSI}

El sistema de prueba es mostrado en Fig. 1. El estado del semiconductor superior de la fase $x$ se define como $S_{x}$, así:

$$
S_{x}=\left\{\begin{array}{l}
1, \quad \text { si } S_{x 1}=O N \\
0, \quad \text { si } S_{x 1}=O F F
\end{array}\right.
$$

$x \in\{a, b, c\}$. Note que $S_{x 2}=\overline{S_{x 1}}$. Ahora, el vector de conmutación del convertidor VSI $\vec{S}=\left(S_{a}, S_{b}, S_{c}\right) \in \boldsymbol{S}_{8}$, donde $\boldsymbol{S}_{8}$ hace mención a un conjunto de 8 elementos que son los posibles vectores de conmutación.

$$
\boldsymbol{S}_{8}=\{000,100,110,010,011,001,101,111\}
$$

\section{B. Ecuaciones de predicción}

Para predecir la corriente $i(k+1)$ se requiere un modelo matemático del sistema. La ecuación discreta que modela el convertidor VSI mostrado en Fig. 1 es (3), donde $\boldsymbol{v}(\boldsymbol{k})$ es el voltaje en las ramas del convertidor en el instante $k$ y $T_{s}$ es el período de muestreo. Reemplazando la derivada discreta (4) y resolviendo, se obtiene la ecuación de predicción de la corriente $\left(i^{P}(k+1)\right)$.

$$
\begin{gathered}
\boldsymbol{v}(\boldsymbol{k})=R \boldsymbol{i}(\boldsymbol{k})+L \frac{d \boldsymbol{i}(\boldsymbol{k})}{d t} \\
\frac{d \boldsymbol{i}(\boldsymbol{k})}{d t}=\frac{\boldsymbol{i}(\boldsymbol{k}+\mathbf{1})-\boldsymbol{i}(\boldsymbol{k})}{T_{s}} \\
\boldsymbol{i}^{\boldsymbol{P}}(\boldsymbol{k}+\mathbf{1})=\left(1-\frac{R}{L} T_{s}\right) \boldsymbol{i}(\boldsymbol{k})+\frac{T_{s}}{L} \boldsymbol{v}(\boldsymbol{k})
\end{gathered}
$$

\section{Algoritmo de COntrol}

Generalmente, la corriente de salida del convertidor VSI es la variable controlada. Fig. 2 muestra un esquema básico del algoritmo de control de corriente predictivo. Las entradas del controlador predictivo son la corriente de referencia $i^{*}$, la corriente medida $\boldsymbol{i}(\boldsymbol{k})$ y el voltaje del bus dc $\left(V_{d c}\right)$. Mientras que el vector de conmutación aplicado en el siguiente período de muestreo $S(k+1)$ es la salida del controlador.

El control de corriente predictivo se realiza en el marco de referencia $\alpha \beta$. En primer lugar, la referencia y las corrientes medidas se transforman a coordenadas $\alpha \beta$. Luego, se predice el valor futuro de la corriente y se calcula la función de costo para cada estado de conmutación. Finalmente, se aplica el estado que minimiza la función de costo. En Fig. 3 se muestra el diagrama de bloques del control de corriente predictivo.

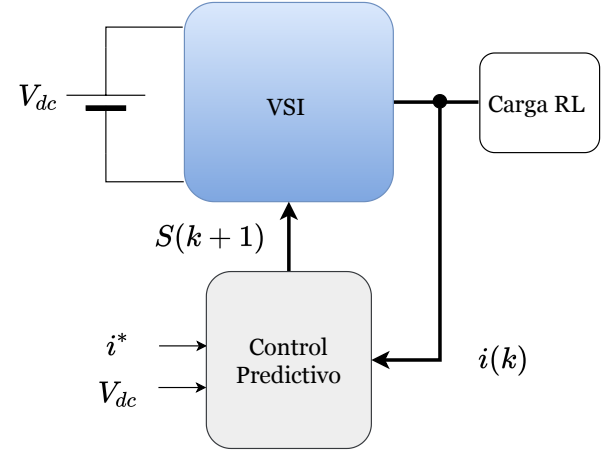

Fig. 2. Esquema general de la ley de control.

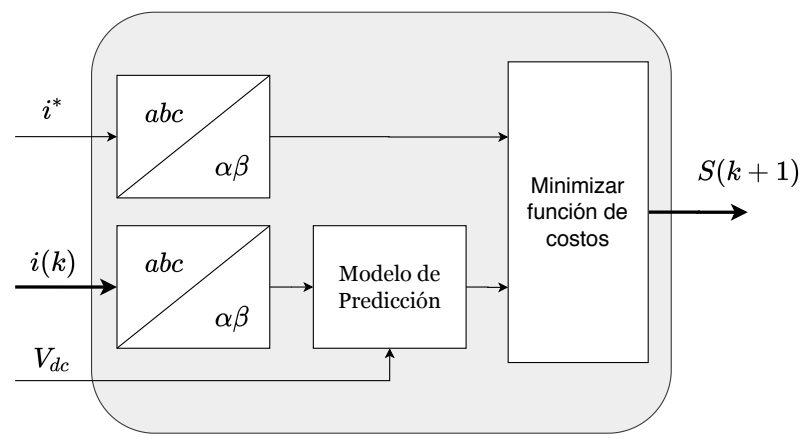

Fig. 3. Diagrama de bloques del controlador predictivo de corriente.

\section{A. Control predictivo con función de costo mono-objetivo}

En el control de corriente predictivo, se evalúa el error de seguimiento de la corriente de salida del convertidor VSI con respecto al valor de referencia. El error de seguimiento se evalúa en la función de costo usando el marco de referencia $\alpha \beta$ de acuerdo a (6).

$$
J_{i}=\left|i_{\alpha}^{*}-i_{\alpha}^{P}(k+1)\right|+\left|i_{\beta}^{*}-i_{\beta}^{P}(k+1)\right|
$$

\section{B. Control predictivo con función de costo multi-objetivo}

Una función de costo multi-objetivo evalúa una función de costo que depende de varios objetivos de control. En la definición de la función de costo global $J$, es necesario incluir factores de peso $(\lambda)$. El caso más simple es la función de costo con dos objetivos de control como (7), donde la primera función de costo $J_{1}$ depende del objetivo principal y la segunda función $J_{2}$ depende del objetivo secundario. En este trabajo, la primera función de costo es $J_{i}$ (ecuación (6)) y la segunda es $J_{v}(9)$.

$$
\begin{aligned}
& J=J_{1}+\lambda J_{2} \\
& J=J_{i}+\lambda J_{v}
\end{aligned}
$$

La ecuación (9) que depende del voltaje del bus dc y del estado del interruptor superior del VSI, establece un voltaje proporcional al voltaje de modo común $(\mathrm{Vcm})$. Con esta definición se entiende que el objetivo principal de control es el seguimiento de corriente y el objetivo secundario es la reducción de $V \mathrm{~cm}$. La función global de costo se muestra en la ecuación (10). 


$$
\begin{gathered}
J_{v}=V_{d c}\left(S_{a}+S_{b}+S_{c}\right) \\
J=\left|i_{\alpha}^{*}-i_{\alpha}^{P}(k+1)\right|+\left|i_{\beta}^{*}-i_{\beta}^{P}(k+1)\right|+\lambda V_{d c}\left(S_{a}+S_{b}+S_{c}\right)
\end{gathered}
$$

\section{Control predictivo secuencial}

En este trabajo se evalúan dos funciones de costo simples de forma secuencial para encontrar el siguiente estado de conmutación. Para la primera función de costo, se evalúan todos los estados posibles del VSI (8 estados). Luego, se eligen los $N$ estados que mejor minimizan la primera función de costo $(N \leq 8)$ para evaluar la segunda función. Finalmente, se aplica el estado que minimiza la segunda función de costo. $N$ es un parámetro de SPC, que representa el número de estados de conmutación que se evaluarán en la segunda función de costo.

Generalmente, el objetivo de control en un VSI es el seguimiento de la corriente de salida. Por esta razón, es común evaluar primero la función de costo de corriente y evaluar después la función de costo que minimiza $\mathrm{Vcm}$. Otra opción es invertir el orden de evaluación de las funciones de costos. Las descripciones de ambos algoritmos SPC se muestran a continuación.
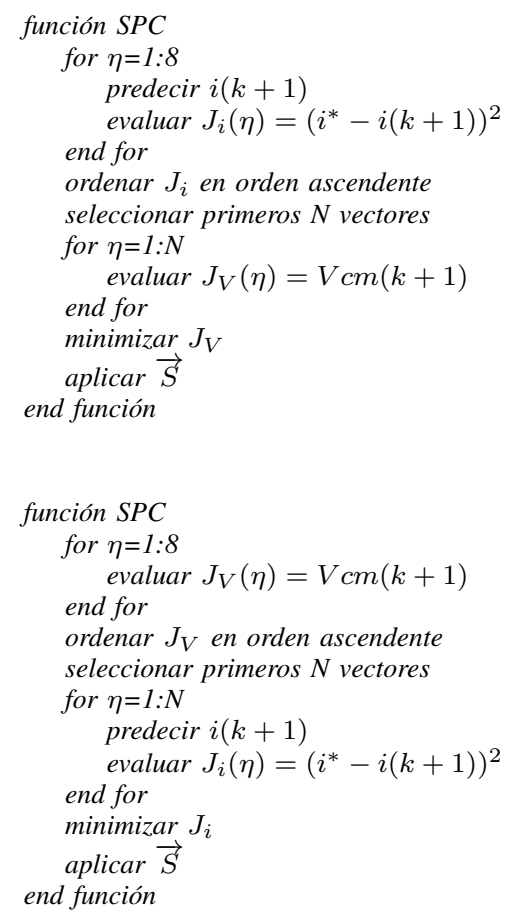

Para implementar el algoritmo de control se debe elegir el valor de $N . N$ es un número entero que satisface la condición $1 \leqslant N \leqslant 8$, siendo 8 el número total de estados posibles. Un valor pequeño de $N$ significa que los estados que mejor minimizan la primera función de costo serán evaluados en la segunda. Por otro lado, si $N$ es grande, significa que los estados mal evaluados en la primera función se evaluarán en la segunda función de costo. Una implicación equivalente es que el valor $N$ define cuál de los objetivos de control tiene la mayor prioridad. Si $N$ es pequeño, la prioridad es el primer
TABLA I

PARÁmetros DEl SISTEMA DE PRUEBA

\begin{tabular}{cc}
\hline Parámetros & Valor \\
\hline$T_{\text {step }}$ & $1 \mu \mathrm{s}$ \\
$T_{s}$ & $20 \mu \mathrm{s}$ \\
$f$ & $60 \mathrm{~Hz}$ \\
$V_{d c}$ & $440 \mathrm{~V}$ \\
$R$ & $0.2 \Omega$ \\
$L$ & $6.3 \mathrm{mH}$ \\
\hline
\end{tabular}
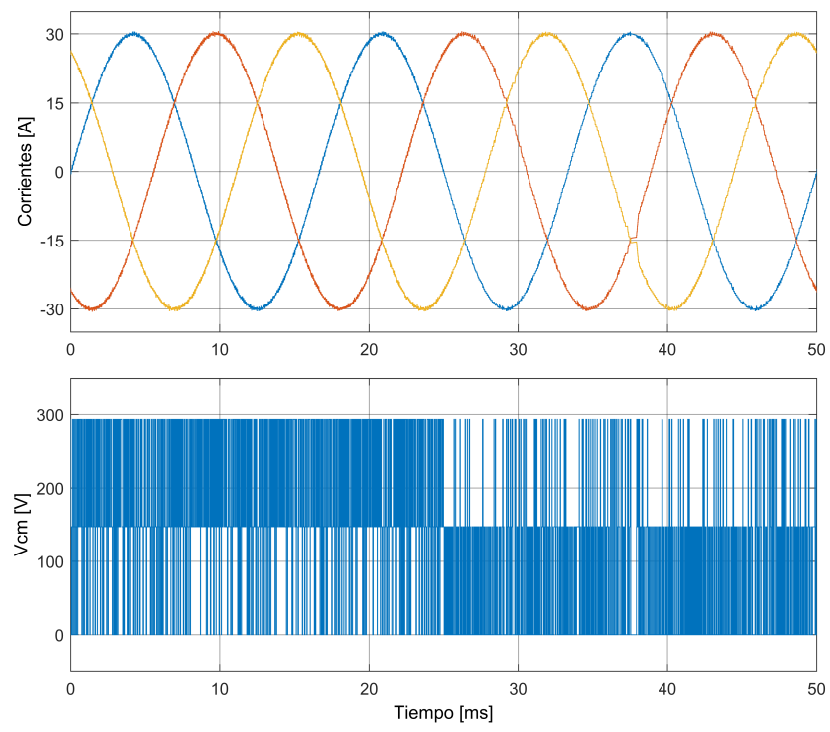

Fig. 4. Resulados del CPC.

objetivo de control y si $N$ es grande, la prioridad es el segundo objetivo. Finalmente, el valor de $N$ debe seleccionarse de acuerdo con los criterios de desempeño establecidos para la aplicación. De hecho si hay varias funciones de costos, es natural querer obtener un balance entre los diferentes objetivos de control.

\section{RESUlTAdos DE SIMULACióN}

En esta sección se presentan los resultados de simulación obtenidos en Matlab/Simulink de los controladores predictivos discutidos anteriormente. Formas de onda de corriente y $\mathrm{Vcm}$ son presentadas en cada caso. Los parámetros del sistema de prueba se incluyen en la Tabla I. El valor de la referencia de corriente es $30 \mathrm{~A}$.

\section{A. Control predictivo clásico}

Los resultados para el control predictivo multi-objetivo considerando seguimiento de corriente y reducción de $\mathrm{Vcm}$ se muestran en Fig. 4. La simulación comienza con un factor de peso $\lambda=0$, valor que cambia a la mitad de la simulación a $\lambda=0,001$. Por lo tanto, para el intervalo $t<25 \mathrm{~ms}$, la función de costo del controlador es mono-objetivo y el objetivo de control es la corriente. Para el intervalo final $t>25 \mathrm{~ms}$, la función de costo es multi-objetivo y los objetivos de control son corriente y $\mathrm{Vcm}$. Los resultados del análisis muestran un buen seguimiento de corriente y $\mathrm{Vcm}$ con un valor máximo de $293 \mathrm{~V}$ en ambos intervalos. 

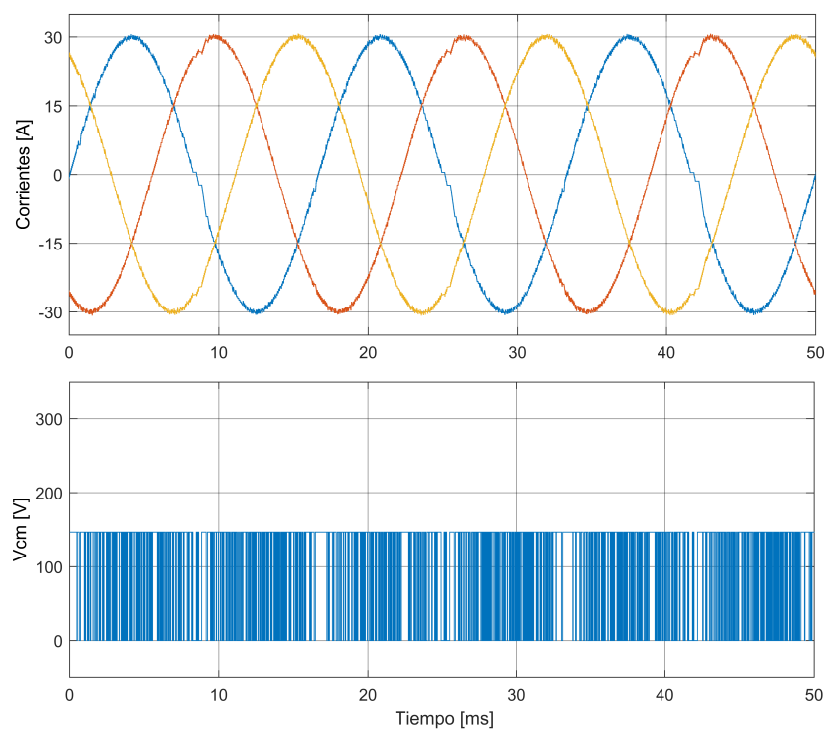

Fig. 5. Resultados del SPC-Caso 1: primero se evalúa la función de costo de corriente y luego el voltaje en modo común.

\section{B. Control predictivo secuencial}

En Fig. 5 se muestran las formas de onda de la corriente y $V \mathrm{~cm}$. Estos resultados se obtuvieron evaluando primero la función de costo de la corriente y luego el $\mathrm{Vcm}$. En Fig. 6 se muestran los resultados obtenidos cambiando el orden de evaluación de las funciones de costo. Los valores de $N$ fueron 2 y 4 respectivamente. Se puede observar en ambos casos que hay un buen seguimiento de corriente con un valor máximo de Vcm de $146.5 \mathrm{~V}$.

\section{DISCUSIÓN}

\section{A. Control predictivo clásico}

Los resultados mostrados en Fig. 4 corresponden a dos valores de $\lambda$ diferentes: $\lambda=0$ para el intervalo $t<25 \mathrm{~ms}$ y $\lambda=0,001$ para el intervalo $t>25$ ms. Debido a que las formas de onda de la corriente en cada intervalo son similares, se incluye el análisis comparativo de contenido armónico en la Tabla II. Se observa en ambos casos que el contenido armónico es bajo. En el primer intervalo donde $\lambda=0$ el índice THD es $1.01 \%$. En el segundo intervalo $\lambda=0.001$ se obtuvo el THD mínimo (0.87\%). Respecto a los resultados de $\mathrm{Vcm}$ mostrados en Fig. 4, es posible identificar tres valores representativos: valor mínimo $0 \mathrm{~V}$, valor intermedio $146.5 \mathrm{~V}$ y valor máximo $293 \mathrm{~V}$. El $\mathrm{Vcm}$ oscila entre estos tres valores en cada intervalo. Para $\lambda=0, V c m$ oscila principalmente entre el valor intermedio y el valor máximo. En el intervalo final $(\lambda=0.001), V c m$ oscila principalmente entre el valor mínimo y el valor intermedio. Además, se puede notar que hay un número menor de transiciones en el segundo intervalo, lo que resulta en una menor distorsión armónica como se verificó en el análisis THD.

\section{B. Control predictico secuencial}

1) Orden de evaluación de las funciones de costo: el orden de evaluación se define de acuerdo a la prioridad de los
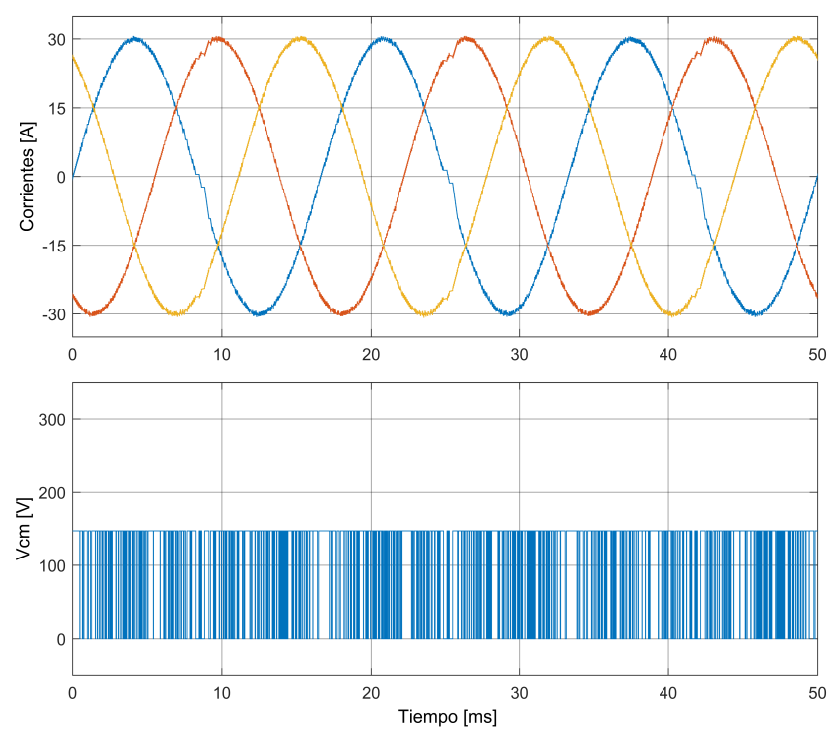

Fig. 6. Resultados del SPC-Caso 2: primero se evalúa el voltaje en modo común y luego la función de costo de corriente.

objetivos de control, donde el objetivo principal se evalúa en la primera función de costo y a continuación se evalúa la segunda función de costo que depende del objetivo de control secundario. Es posible intercambiar el orden de las funciones de costo y obtener resultados satisfactorios como se muestra en los resultados anteriores (ver Figs. 5 y 6).

2) Dominio de los parámetros de control: $N$ es un parámetro del SPC. $N$ es un número entero que representa el número de estados de conmutación que se evaluarán en la segunda función de costo. Los posibles valores de $N$ dependen del orden de evaluación de las funciones de costo. Hay dos casos:

- Caso 1: primero se evalúa la función de costo de corriente y luego el voltaje en modo común.

- Caso 2: primero se evalúa el voltaje en modo común y luego la función de costo de corriente.

Es posible definir mediante estudios de simulación un intervalo de selección para el parámetro $N$.

- Caso 1: $N \in[2,3]$ ( $N=2$ en los resultados mostrados en Fig. 5).

- Caso 2: $N \in[4,6]$ ( $N=4$ en los resultados de Fig. 6).

Para cada valor de $N$ de los intervalos anteriores, se obtienen resultados satisfactorios considerando ambos objetivos de control. Satisfactorio significa que la corriente de carga tiene forma de onda sinusoidal y que hay una reducción de $V c m$. Los valores mínimos que puede tomar $N$ son 2 en el caso I y 4 en el caso II. Los valores máximos son 3 y 6 respectivamente.

3) Desempeño del SPC de acuerdo a los parámetros elegidos: se selecciona el caso II para el análisis de desempeño del SPC bajo diferentes valores de $N$. En este caso, el objetivo principal es reducir $V c m$ y el objetivo secundario es el seguimiento de corriente. Fig. 7 muestra gráficos de $V c m$ para los tres posibles valores de $N$. El mejor desempeño se obtiene para $N=4$ que es el valor más bajo posible. 

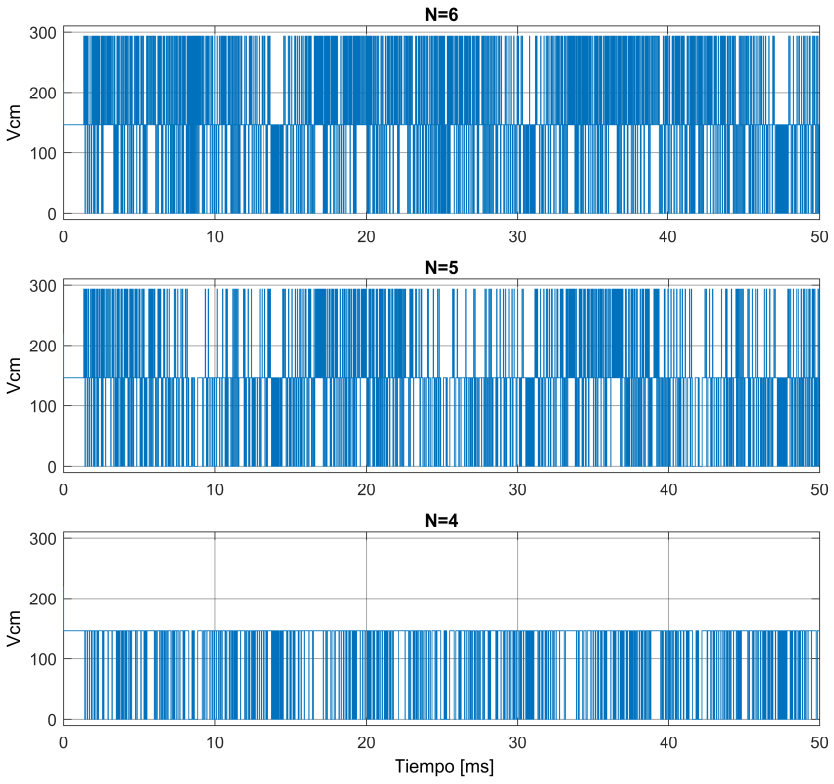

Fig. 7. Desempeño del SPC para diferentes valores de $N$.
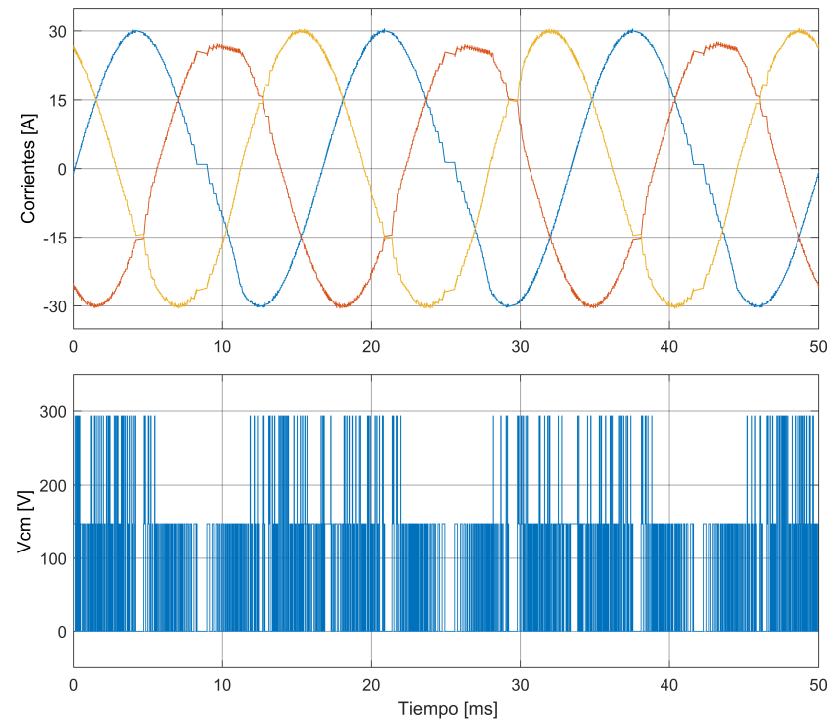

Fig. 8. Desempeño CPC para $\lambda=0.0013$.

Para valores de $N$ superiores, el valor máximo de $V c m$ y la frecuencia de conmutación aumentan. Para valores inferiores el comportamiento es opuesto. La distorsión armónica más baja es $1,18 \%$ obtenida para $N=6$. Los otros índices THD son $2,32 \%(N=4)$ y $1,53 \%(N=5)$. Se concluye que valores pequeños de $N$ dan como resultado $\mathrm{Vcm}$ más bajo y un índice THD más alto. Para valores mayores de $N$ el desempeño es contrario.

\section{Comparación entre CPC y SPC}

1) Voltaje en modo común: se puede observar en Figs. 4, 5 y 6, que los dos controladores tienen desempeños muy diferentes respecto al $\mathrm{Vcm}$. La observación más importante es que en CPC el valor máximo de $\mathrm{Vcm}$ permanece constante en
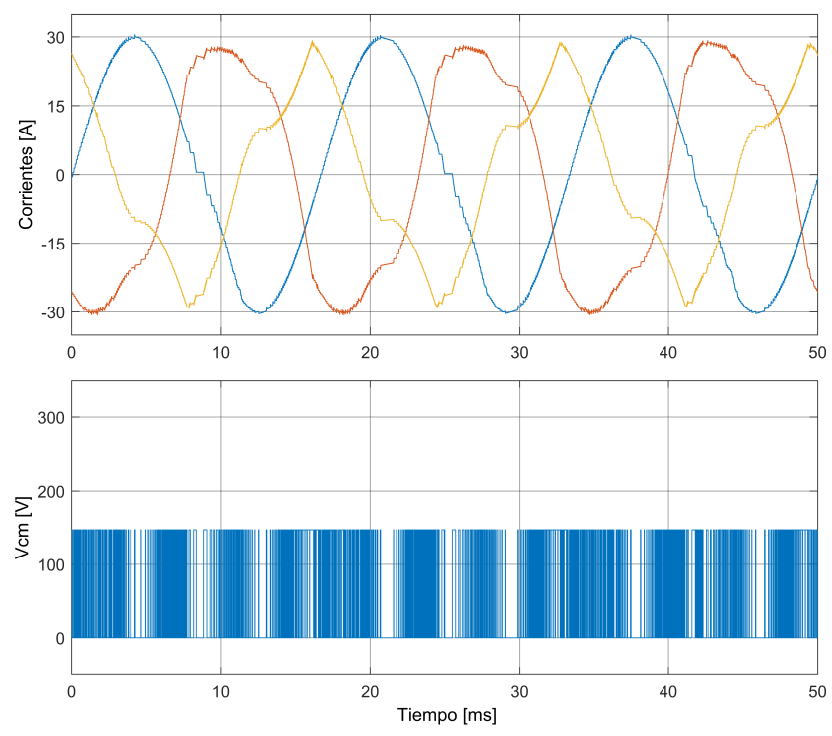

Fig. 9. Desempeño CPC para $\lambda=0.0015$.

TABLA II

ANÁLISIS DEL CONTENIDO ARMÓNICO EN CPC

\begin{tabular}{lcc}
\hline$\lambda$ & 0 & 0.001 \\
\hline Fundamental [Ap] & 30.00 & 30.01 \\
Fundamental [Arms] & 21.21 & 21.22 \\
THD [\%] & 1.01 & 0.87 \\
Componente DC [\%] & 0.04 & 0.03 \\
Principales armónicos & $360 \mathrm{~Hz} 0.06 \%$ & $180 \mathrm{~Hz} 0.09 \%$ \\
& $420 \mathrm{~Hz} 0.06 \%$ & $540 \mathrm{~Hz} 0.05 \%$ \\
& $600 \mathrm{~Hz} 0.06 \%$ & $360 \mathrm{~Hz} 0.04 \%$ \\
\hline
\end{tabular}

TABLA III

ANÁLISIS DEL CONTENIDO ARMÓNICO EN SPC

\begin{tabular}{lcc}
\hline Caso & Caso 1 & Caso 2 \\
\hline Fundamental [Ap] & 29.98 & 29.99 \\
Fundamental [Arms] & 21.20 & 21.20 \\
THD [\%] & 2.36 & 2.32 \\
Componente DC [\%] & 0.19 & 0.26 \\
Principales armónicos & $180 \mathrm{~Hz} 0.60 \%$ & $120 \mathrm{~Hz} \mathrm{0.63 \%}$ \\
& $120 \mathrm{~Hz} 0.51 \%$ & $240 \mathrm{~Hz} 0.55 \%$ \\
& $480 \mathrm{~Hz} 0.51 \%$ & $360 \mathrm{~Hz} 0.44 \%$ \\
\hline
\end{tabular}

$293 \mathrm{~V}$, mientras que en SPC este valor se reduce a la mitad $(146.5 \mathrm{~V})$. Se debe considerar que en los resultados de CPC presentados en Fig. 4, el factor de peso utilizado en el segundo intervalo de simulación ( 25 - $50 \mathrm{~ms}$ ) fue $\lambda=0.001$. Con este valor se obtuvo un balance entre los dos objetivos de control. Esto significa un buen seguimiento de corriente y un mejor desempeño del $\mathrm{Vcm}$ como se mencionó anteriormente, pero no hay reducción en el valor máximo de $\mathrm{Vcm}$. De acuerdo con la función de costo propuesta, si se aumenta el factor de costo se minimiza el $\mathrm{Vcm}$. Este afirmación se valida en Figs. 8 y 9 , donde se muestran los resultados de corriente y $\mathrm{Vcm}$ para los factores de peso 0,0013 y 0,0015 respectivamente. Estos resultados sugieren que no es posible reducir $\mathrm{Vcm}$ a un valor de $146.5 \mathrm{~V}$ sin afectar el seguimiento de corriente. Se concluye que CPC presenta limitaciones para establecer un balance entre los dos objetivos de control. 
TABLA IV

ANÁLISIS DEL CONTENIDO ARMÓNICO DEL CPC

\begin{tabular}{ccc}
\hline THD & $\lambda=0.0013$ & $\lambda=0.0015$ \\
\hline Fase a & 8.01 & 6.19 \\
Fase b & 5.19 & 16.26 \\
Fase c & 4.87 & 21.53 \\
\hline
\end{tabular}

2) Contenido armónico: los resultados mostrados en Figs. 4,5 y 6 se caracterizan por un buen seguimiento de la corriente. Resultados previos indican que el índice THD obtenido para CPC es menor que para el SPC. El valor THD correspondiente en CPC para $\lambda=0.01$ fue $0.87 \%$, mientras que para la técnica SPC el THD es aproximadamente $2.3 \%$. Debido a que los resultados del controlador CPC no fueron satisfactorios en términos de reducción de $V c m$, se realizaron análisis adicionales para otros factores de peso $(\lambda=0.0013$ y $\lambda=0.0015)$ como se muestra en Figs. 8 y 9. El aumento del factor de peso en la función de costo de CPC mejora la reducción de $\mathrm{Vcm}$ pero introduce una distorsión armónica significativa y desbalance de fases. El análisis armónico por fase para cada factor de peso se presenta en la Tabla IV.

\section{Principales hallazgos}

Los principales hallazgos obtenidos en esta investigación son:

- Reducir el voltaje en modo común en la función de costo, contribuye a reducir el contenido de armónicos en las corrientes de salida del VSI.

- Es posible cambiar el orden de evaluación de las funciones de costo en SPC, pero el parámetro $N$ debe elegirse apropiadamente.

- SPC presenta un mejor rendimiento en la reducción de voltaje en modo común en comparación con CPC.

- Menores valores del parámetro $N$ resultan en minimizar el primer objetivo de control y maximizar el segundo. Valores mayores de $N$ producen resultados opuestos.

- En SPC debe haber un balance entre los objetivos de control. Los criterios de desempeño son específicos de la aplicación.

- En CPC es complejo establecer un balance entre los objetivos de control, lo que si se logra fácilmente con la técnica SPC.

\section{CONCLUSIONES}

En este trabajo se utilizó la técnica SPC en el seguimiento de corriente y la reducción de voltaje en modo común en un sistema VSI con carga RL. Se realizaron análisis del orden de evaluación de las funciones de costo y los posibles valores del parámetro de control $N$. Este parámetro debe elegirse adecuadamente para obtener un buen desempeño del controlador SPC respecto a ambos objetivos de control. Los mejores resultados para reducir el voltaje en modo común se obtuvieron evaluando primero el voltaje en modo común y luego la función de costo de corriente. En este caso, el valor máximo de $\mathrm{Vcm}$ se redujo en $50 \%$ obteniendo un índice THD para la corriente de carga de $2,32 \%$. El valor mínimo del THD fue 1,18\% pero el $\mathrm{Vcm}$ alcanzó el valor máximo.
En este trabajo no fue posible reducir el valor máximo del voltaje de modo común en $50 \%$ usando CPC y aún así obtener un sistema de corrientes balanceadas con un THD permisible. En cambio, este desempeño si se obtuvo con SPC. Los resultados muestran que con la técnica CPC es complejo obtener un balance entre los objetivos de control, lo que si se logró fácilmente con la técnica SPC.

\section{AgRAdECIMIENTOS \\ Este trabajo fue financiado por el Gobierno de Chile bajo los proyectos ANID/FONDECYT/1191028, ANID/FONDECYT/1191680, ANID-PFECHA/Doctorado-Nacional/2019- \\ 21191663, ANID/FONDECYT/MEC80190074 y ANID/FONDECYT/MEC80180097. El trabajo también fue financiado por SERC Chile (ANID/FONDAP/15110019).}

\section{REFERENCIAS}

[1] X. Wang, J. Zhao, Q. Wang, G. Li, and M. Zhang, "Fast fcs-mpc-based svpwm method to reduce switching states of multilevel cascaded hbridge statcoms," Journal of Power Electronics, vol. 19, no. 1, pp. 244253, 2019.

[2] D. Xiao, K. S. Alam, M. Norambuena, M. F. Rahman, and J. Rodriguez, "Modified modulated model predictive control strategy for a gridconnected converter," IEEE Transactions on Industrial Electronics, pp. $1-1,2020$.

[3] S. Borreggine, V. G. Monopoli, G. Rizzello, D. Naso, F. Cupertino, and R. Consoletti, "A review on model predictive control and its applications in power electronics," in 2019 AEIT International Conference of Electrical and Electronic Technologies for Automotive (AEIT AUTOMOTIVE), 2019, pp. 1-6.

[4] Y. Yang, H. Wen, M. Fan, M. Xie, and R. Chen, "Fast finite-switchingstate model predictive control method without weighting factors for ttype three-level three-phase inverters," IEEE Transactions on Industrial Informatics, vol. 15, no. 3, pp. 1298-1310, 2018.

[5] Y. Yang, H. Wen, M. Fan, M. Xie, R. Chen, and Y. Wang, "A constant switching frequency model predictive control without weighting factors for t-type single-phase three-level inverters," IEEE Transactions on Industrial Electronics, vol. 66, no. 7, pp. 5153-5164, 2018.

[6] R. E. Pérez-Guzmán, M. Rivera, and P. W. Wheeler, "Weighting factor design in model predictive control for power converters," in 2019 IEEE CHILEAN Conference on Electrical, Electronics Engineering, Information and Communication Technologies (CHILECON), 2019, pp. $1-6$.

[7] J. Zhang, L. Li, M. Norambuena, J. Rodriguez, and D. G. Dorrell, "Sequential model predictive control of direct matrix converter without weighting factors," in IECON 2018-44th Annual Conference of the IEEE Industrial Electronics Society. IEEE, 2018, pp. 1477-1482.

[8] M. Norambuena, J. Rodriguez, Z. Zhang, F. Wang, C. Garcia, and R. Kennel, "A very simple strategy for high-quality performance of ac machines using model predictive control," IEEE Transactions on Power Electronics, vol. 34, no. 1, pp. 794-800, Jan 2019.

[9] Y. Li, Z. Zhang, and M. P. Kamierkowski, "Cascaded predictive control for three-level npc power converter fed induction machine drives without weighting factors," in 2018 IEEE International Power Electronics and Application Conference and Exposition (PEAC). IEEE, 2018, pp. 1-5.

[10] Y. Wang, Z. Zhang, W. Huang, R. Kennel, W. Xie, and F. Wang, "Encoderless sequential predictive torque control with smo of 31-npc converter-fed induction motor drives for electrical car applications."

[11] S. A. Davari, M. Norambuena, V. Nekoukar, C. Garcia, and J. Rodriguez, "Even-handed sequential predictive torque and flux control," IEEE Transactions on Industrial Electronics, 2019.

[12] M. S. Mousavi, S. A. Davari, V. Nekoukar, M. Norambuena, J. Rodriguez, and F. Wang, "Four-stage cascaded predictive control for zerosequence current suppression of open-end winding induction motor," in 2020 11th Power Electronics, Drive Systems, and Technologies Conference (PEDSTC). IEEE, 2020, pp. 1-6. 